\title{
Convergent Points for Conventional Medicine and Whole Systems Research: A User's Guide
}

\author{
Charles Elder, MD, MPH, FACP, Nadine ljaz, PhD, ${ }^{2}$ John Weeks, ${ }^{3}$ \\ Jennifer Rioux, $\mathrm{PhD}^{4}$ and Cheryl Ritenbaugh, $\mathrm{PhD}, \mathrm{MPH}^{5}$
}

\begin{abstract}
Context: Value-based health care has emerged as a manifestation of the conventional medicine community's awareness of the overlapping needs to both better incorporate patient centeredness into practice and research paradigms and further develop a systemic approach to cost reduction.

Background: The origins of the whole systems research (WSR) movement date to the late 1990s, when the U.S. Congress legislated appropriation of funds to stimulate the U.S. National Institutes of Health to evaluate popular traditional, complementary, and integrative medicine (TCIM) practices. Questions immediately arose over how well these forms of practice could be measured through standard randomized controlled trials, and the WSR community began to articulate and adapt innovative methodologies for evaluating TCIM interventions.

Discussion: This column explores the potential impact of WSR methods and exemplars on the clinical practice and research communities seeking to successfully implement and measure the complexities of valuebased health care. Four potentially cross-talking themes are specifically discussed: complex behaviorally focused interventions, patient-centered outcomes, team-based care, and resilience and well-being.

Conclusion: The time is ripe for clinicians and investigators to capitalize on methodologies, exemplars, and learnings from the WSR literature toward improving care, developing more robust research strategies, and furthering the dialogue between the TCIM and conventional medicine communities.
\end{abstract}

Keywords: whole systems research, value-based health care, integrative medicine

\section{Introduction}

A CENTRAL FOCUS OF THIS special issue is the exploration of the convergence of two movements in health care. The introductory editorial speaks of these two streams of activity. One is the relatively recent paradigm shift from volume to value - including "value-based health care" along with "value-based payment." 1 The emphasis, as distinct from production of services, is in promoting the goals of the "Quadruple Aim': enhancing patient experience, improving practitioner satisfaction, bettering population health, and lowering per capita costs. Paramount concerns within valuebased health care include management of chronic disease, addressing behavioral and other determinants of health, interprofessionalism and team care, patient centeredness and engagement, effectiveness rather than just efficacy, and a growing interest in fostering resilience and well-being.

The second stream of activity relates to the evaluation of traditional, complementary, and integrative medicine (TCIM) practices. The echoes between value-based care and this earlier movement in U.S. medicine are intriguing. In 1998, the U.S. Congress legislated appropriation of funds to stimulate the U.S. National Institutes of Health to research the "modalities, systems, and disciplines" linked to the popular use TCIM practices. ${ }^{2}$ Highlighted as a practical aim was "the integration of alternative treatment, diagnostic and prevention

\footnotetext{
${ }^{1}$ Kaiser Permanente Center for Health Research, Portland, OR.

${ }^{2}$ Leslie Dan Faculty of Pharmacy, University of Toronto, Toronto, Canada.

${ }^{3}$ johnweeks-integrator.com, Editor-in-Chief, JACM, Seattle, WA.

${ }^{4}$ Integral Ayurveda and Yoga Therapy, Carrboro, NC.

${ }^{5}$ Family and Community Medicine, University of Arizona, Tucson, AZ.
} 
systems, modalities, and disciplines with the practice of conventional medicine as a complement to such medicine and into health care delivery systems." Questions immediately arose over how well these forms of practice could be measured through standard single agent placebo-controlled randomized trials. Many TCIM approaches are typically multimodal, individually tailored, holistic, often team-based, and focused on healing rather than reduction of the indices of disease. Researchers associated with these communities of TCIM practice began to articulate and adapt innovative methodologies. They called it "whole systems research" (WSR).

This column was invited to explore the extent to which these WSR methods and research exemplars may be useful to the clinical practice and research communities seeking to successfully implement and measure the complexities of value-based health care. To do so, the authors have relied on the review work led by Ijaz et al. and reported in this issue. The authors chose to arrange their inquiry around four potentially cross-talking themes: multimodality or complex behaviorally focused interventions; patient-centered outcomes; team-based care; and resilience and well-being. Although not exhaustive, this review is intended as a practical users' guide for those unfamiliar with WSR. The aim is to provide an introductory manual for connecting practitioners, administrators, investigators, and other stakeholders to the WSR literature in a pragmatic way that can illuminate and enhance the evaluation and delivery of care based on these emerging considerations and objectives.

\section{Complex Behaviorally Focused Interventions}

Clinically, the conventional medicine community has recognized the importance of complex behaviorally focused interventions for the optimal management of complex chronic diseases. In one landmark study, Ornish et al. ${ }^{3}$ randomly assigned individuals with coronary artery disease to a standardized multimodality behavioral intervention, delivered in a group setting, compared with control. The experimental intervention consisted of a low-fat vegetarian diet, smoking cessation, stress management, and exercise. After 1 year, angiographically assessed coronary artery stenosis regressed in the experimental group, while progressing in the control group. These are remarkable results from an entirely noninvasive intervention.

Various approaches can be taken to evaluate complex interventions, ranging from establishing a single standardized protocol for use by all patients, as in the Ornish article ${ }^{3}$ to allowing full practitioner discretion in selecting modalities and individualizing treatment (see Figure 2, Ijaz et al.). The importance of individually tailoring approaches is evident in emerging conventional models that respect consideration of an individual's readiness for change and the clarification of purpose that may drive change behavior. In the Diabetes Prevention Program, the behavioral intervention ${ }^{4}$ included a core curriculum that provided for standardization. At the same time, individualization could be accomplished through a toolbox of adherence strategies. Likewise, case managers were able to adapt the intervention to the needs of specific ethnic populations.

The WSR literature extends this concept by evaluating treatment individualization within a clinical trial based on clinical subtypes, such as a Traditional Chinese Medicine (TCM) diagnosis. For example, Ritenbaugh et al. ${ }^{5}$ modeled a manualization with tailoring approach that allowed for protocol-driven individualization. One hundred sixty-eight participants with temporomandibular joint dysfunction (TMD) attended a basic TMD class introducing the fundamentals of self-care. Then, at weeks 2 and 10, participants who continued to report facial pain above threshold levels were allocated to either continued conventional specialty care or TCM. TCM clinicians had the flexibility to deliver interventions that included not only acupuncture but also moxibustion, Chinese herbs, massage (Tuina), and lifestyle and nutrition counseling. For acupuncture administration, a core set of points was prespecified, but could be supplemented per protocol by the acupuncturist to address individual patient needs. Likewise, for herbal therapy, a base herbal formula was specified for each of 12 TCM diagnoses, but practitioners had flexibility within the protocol to individualize to the patient. The investigators showed that whole system, individualized TCM, provided significantly greater short-term pain relief along with significant reductions in social interference. Another randomized controlled trial, by Kessler et al., ${ }^{6}$ employed a similar strategy, this time offering whole system ayurvedic therapies compared with usual care for patients with osteoarthritis of the knee.

Allopathic clinicians require a broader toolbox to more effectively manage chronic musculoskeletal pain; where there is generally no biomarker, diagnostic categories are often overlapping or vague, and standard medical therapies may be toxic, addictive, or ineffective. The manualization with tailoring approach (see Figure 2, Ijaz et al.), as exemplified in the mentioned WSR examples, thus holds potential for substantially enhancing chronic musculoskeletal pain management. Specifically, clinical trials of complex TCIM interventions conceivably could extend the pain management evidence base through expanded clinical protocols drawing from both conventional and TCIM approaches. ${ }^{7}$ The addition of Chinese medicine or ayurvedic diagnostic differentiation within a pain management study offers potential to identify additional clinically relevant categories of patients for whom diagnostically specified targeted therapies could further improve outcomes. This, in turn, could contribute to dissemination of better nonpharmacologic options for managing chronic musculoskeletal pain.

Investigators could likewise extend this thinking to other chronic conditions within conventional medicine. For example, in evaluating behavioral approaches to managing obesity, diabetes, or cardiovascular disease, investigators might allow for a range of dietary and lifestyle options in a multimodality cardiovascular or obesity study. Such interventions could be personalized based on patient preference (for one from a range of diets), comorbid conditions (depression, chronic pain, obstructive lung disease, etc.), genomics, change readiness, or other factors. This could enhance the range of appeal of an intervention, and thus increase the external validity of findings and facilitate dissemination. Studies by Bredesen et al., ${ }^{8}$ Cooley et al., ${ }^{9}$ Ritenbaugh et al., ${ }^{10}$ Hullender Rubin et al., ${ }^{11}$ Rioux et al., ${ }^{12}$ Wang et al., ${ }^{13}$ and others (see Ijaz et al.) provide additional examples of the assessment of complex whole systems interventions in the setting of dementia, diabetes, chronic pain, infertility, obesity, mental health conditions, and other disorders. 


\section{Patient-Centered Outcomes}

Patient-centered outcomes of care ${ }^{14}$ remain a focus of the value-based health care movement. ${ }^{1}$ Supplementing biomarkers with patient-reported measures can help to better inform patients, clinicians, and the care team regarding treatment alternatives and impact. Although multimodal approaches often puzzle those researchers and clinicians who seek to isolate a single active ingredient, patient-reported outcomes can help capture the effects of a complex intervention across multiple domains.

An example where examination of such outcomes may be instructive is in the evaluation of obesity and frailty. Many frail older adults are too thin, yet there is also evidence that obesity contributes to frailty in elderly populations. Porter Starr et al. ${ }^{15}$ reviewed randomized controlled trials from the conventional medicine literature related to weight loss and/or exercise interventions for obesity in older adults, with a view toward elucidating the relationship between physical obesity and frailty. Their analysis of 21 trials found strong evidence that weight reduction and exercise interventions improved physical function. However, a complexity of metabolic influences left questions as to which lifestyle interventions impact physical frailty. WSR demonstrates that much can be gained by including patient-reported outcomes in assessing the impact of diet and exercise interventions on frailty in older adults. Specifically, beyond metabolic biomarkers, understanding the impact of such interventions on motivation, mood, self-efficacy, and other parameters could help clarify the role of these interventions in managing both obesity and frailty in the elderly.

For instance, Bradley et al. ${ }^{16}$ described a cohort of 40 patients who received adjunctive whole system naturopathic care in addition to usual care for the management of type 2 diabetes. Adjunctive naturopathic care was individualized, and included recommendations that could encompass diet, exercise, medications, mind-body techniques, and supplements. In addition to documenting the impact of naturopathic care on glycemic control, the investigators collected a range of patient-reported outcomes. They documented improvements not only in blood sugar, but also in multiple patient-reported measures, including glucose testing, diet, physical activity, mood, self-efficacy, and motivation to change lifestyle. Importantly, $63 \%$ of patients reported that they changed the way they think about diabetes as a result of naturopathic care, whereas $72 \%$ reported that they had changed their diabetes care. Clearly, the inclusion of these patient-reported outcomes provided for a much richer and clinically pertinent description of the impact of the complex individualized naturopathic intervention.

The use of patient-reported outcomes can also enhance care by facilitating the rigorous evaluation of complex interventions in clinical contexts, where reliable biomarkers are unavailable. Shalom-Sharabi et al. ${ }^{17}$ used patient-reported outcomes in describing the impact of a tailored TCIM intervention on gastrointestinal symptoms and quality of life in patients with breast and gynecologic cancer. TCIM treatments included supplements, acupuncture, and other manual therapies, and mind-body techniques. Those in the intervention group showed significant improvements in multiple patient-reported measures, including appetite, fatigue, cognitive functioning, emotional functioning, pain, anxiety, and sleep. Likewise, Cooley et al. ${ }^{9}$ demonstrated a statistically significant advantage to multimodality naturopathic care compared with standardized psychotherapy in reducing a range of patient-reported measures, including anxiety.

Finally, publications from the WSR literature by Koithan et al., ${ }^{18}$ Oberg et al., ${ }^{19}$ Ritenbaugh et al., ${ }^{20}$ and Thompson et al. ${ }^{21}$ model the collection and analysis of qualitative data as another important approach for determining and assessing outcomes of importance to participants. One notable qualitative article $^{22}$ described multiple unanticipated benefits commonly reported by patients receiving TCIM therapies, including increased hope, increased ability to relax, improved emotional state, and increased body awareness. Johnson et al. ${ }^{23}$ reported on the successful use of a self-report tool developed from qualitative data to allow participants to self-report on the broad impact of an integrative wellness program. The use of focus groups, structured individual interviews, and other qualitative methods, as a supplement to the routine collection of biomarkers, thus offers opportunity for a deeper understanding of the context and impact of a clinical intervention.

\section{Team-Based Care}

In the biomedical literature, team-based interventions have been shown to be effective for improving the clinical management of chronic medical conditions, such as hypertension. ${ }^{24}$ Whole system TCIM is commonly delivered by teams of practitioners, and the WSR literature contains multiple examples of effective team-based clinical programs. Hamre et al. ${ }^{25}$ described the impact of a complex anthroposophic intervention on low back pain. Anthroposophic care was delivered by a team of clinicians that included not only a physician but also a eurythmy (movement) specialist, a massage therapist, and an art therapist. Participants reported sustained improvements in pain, function, and quality of life. In another example, Attias et al. ${ }^{26}$ reported on the impact of standard anxiolytic therapy with or without TCIM interventions in managing preoperative anxiety. In this case, TCIM providers, including acupuncturists, reflexologists, and others, worked side by side with the surgical care team. The investigators showed that the integration of TCIM services was associated with significant reductions in preoperative anxiety.

In both conventional and TCIM medicine settings, where the patient is receiving care from multiple types of practitioners, a de facto team exists. Understanding the dynamics of how such multidisciplinary teams interact and function can reveal opportunities for improving care delivery and outcomes. Grant and Bensoussan ${ }^{27}$ interviewed leaders and practitioners from a range of integrative health settings in the United States. They found only minimal support for alignment of TCIM delivery within any of the involved clinical centers, and little in the way of formal structure to promote collaboration among TCIM and conventional clinicians. None of the centers provided protocols, guidelines, or ongoing patient monitoring. Joint planning for patient treatment was typically ad hoc and through informal mechanisms only.

In another example, Penney et al. ${ }^{28}$ examined the patterns of communication among the team of people caring for patients receiving acupuncture and chiropractic care at a large health maintenance organization, including primary care doctors, contracted acupuncturists and chiropractors, health plan referral center administrators, and the patients themselves. Focus group and structured interview data were collected in the context of a mixed-methods comparative effectiveness study comparing acupuncture with usual care, and chiropractic care 
with usual care, for patients with chronic musculoskeletal pain. ${ }^{29}$ The qualitative data revealed the care teams to be highly dysfunctional. TCIM clinicians' chart notes never reached the electronic medical record, and the TCIM medicine practitioners and primary care physicians almost never talked to each other, even when they disagreed on the need for an imaging study, or other aspects of the care plan.

Both examples suggest that the most direct route to optimizing outcomes for patients receiving team-based care may have less to do with specifying an active ingredient than with improving the function of the interdisciplinary health care team. Conventional biomedical care is commonly delivered by teams of professionals with different training and perspectives, including primary care physicians, specialists, nurses, medical assistants, behavioral health clinicians, pharmacists, physical therapists, and so on. Focusing research on optimizing teambased interactions and including TCIM as part of interprofessional education curricula ${ }^{30}$ hold potential to improve outcomes across both TCIM and conventional medicine settings.

\section{Resilience and Well-Being}

In the past half decade, a dialogue has ripened among some leaders of large medical delivery organizations about the difference between managing disease and creating health. ${ }^{31-33}$ This parallels the health-orientated paradigm shift that inspired many early WSR researchers to develop improved research models. Patients, too, seek not just disease treatment but also salutogenesis, that is to say, resilience and wellbeing. As evidence of this shift, Gate et al. ${ }^{34}$ piloted an evidence-based psychological intervention to promote behavior change and well-being in patients receiving outpatient clinic care at the Royal Free London NHS Foundation Trust. The wellness intervention consisted of a 4-week evidencebased program that included consultation, motivational support, goal setting, and referrals for mindfulness training and other relevant community services. The investigators found that after 4 weeks, scores for self-efficacy, health, and wellbeing significantly improved, with participants meeting $63 \%$ of lifestyle goals as well as $89 \%$ of health management goals.

The WSR literature extends this concept in several ways. First, the WSR community has published studies wherein resilience or well-being are themselves the focus of diagnosisspecific interventions. In one publication, Witt et al. ${ }^{35}$ reported on results of a pragmatic randomized trial assessing the impact of a complex TCIM intervention for patients with breast cancer. Participants were randomized to receive either usual care alone or usual care plus individualized multimodality TCIM. Such modalities could include supplements, movement therapy, acupuncture, and a range of other choices, with main outcome measures focusing on health-related quality of life. After 6 months, those assigned to the TCIM group showed significantly higher health-related quality of life than control. In another article, Seely et al. ${ }^{36}$ reported on the results of a randomized controlled trial comparing usual care with or without naturopathic care among postal workers at high cardiovascular risk. At 52 weeks, those randomized to receive naturopathic care achieved significant reductions in 10-year cardiovascular risk score, as well as a lower incidence of metabolic syndrome.

Another compelling example from the WSR literature provides for the evaluation of an intensive multimodality wellness intervention delivered to a healthy population. Mills et al. ${ }^{37}$ recruited 69 healthy individuals and allocated them to participate either in a 6-day residential ayurvedic purification and wellness intervention or to simply take a vacation at the same location. The ayurvedic intervention included cleansing and detoxification protocols, a special plant-based diet, daily ayurvedic oil massage treatments, and heating treatments using sauna and/or steam. At 1 month follow-up, participants in the experimental group showed significant improvements in measures of spirituality, gratitude, and self-compassion, as well as reductions in anxiety. Results suggest that a whole systems ayurvedic approach can serve to enhance wellness, even in a healthy population.

In conventional medicine as noted, major health care organizations are beginning to realize they need not wait for a patient to manifest disease, nor even to come to a hospital or clinic. There is opportunity for both enhancing the patient experience and promoting population health through the delivery of wellness-oriented interventions to at risk or healthy populations. The TCIM approaches with which the WSR community is associated are well aligned with this focus on moving beyond symptom reduction and disease management to fostering health and well-being.

\section{Conclusions}

Value-based health care has emerged as a manifestation of the conventional medicine community's awareness of the need to better incorporate the goals of the "Quadruple Aim": enhancing patient experience, improving practitioner satisfaction, bettering population health, and lowering per capita costs. Evaluations of TCIM as published in the WSR literature can help inform the assessment and implementation of value-based care across the range of these shared priorities The time is ripe for clinicians and investigators to capitalize on methodologies, exemplars, and learnings from the WSR literature toward improving care, developing more robust research strategies, and furthering the dialogue between the TCIM and conventional medicine communities. In doing so, we can help fulfill the fundamentally convergent Congressional mandate to the NIH NCCIH to examine the integration of these complex systems and disciplines "with the practice of conventional medicine as a complement to such medicine and into health care delivery systems.",2

\section{Author Disclosure Statement}

No competing financial interests exist.

\section{References}

1. Tsevat J, Moriates C. Value-based health care meets costeffectiveness analysis. Ann Intern Med 2018;5:329-332.

2. The Integrator Blog. Online document at: http://theintegrator blog.com/index.php?option=com_content\&task=view\&id= 606\&Itemid=189, accessed November 25, 2018.

3. Ornish D, Brown SE, Scherwitz LW, et al. Can lifestyle changes reverse coronary heart disease? The Lifestyle Heart Trial. Lancet 1990;336:129-133.

4. Group DPPDR. The Diabetes Prevention Program (DPP): Description of lifestyle intervention. Diabetes Care 2002; 25:2165-2171.

5. Ritenbaugh C, Hammerschlag R, Dworkin SF, et al. Comparative effectiveness of traditional Chinese medicine 
and psychosocial care in the treatment of temporomandibular disorders-associated chronic facial pain. J Pain 2012; 13:1075-1089.

6. Kessler CS, Dhiman KS, Kumar A, et al. Effectiveness of an Ayurveda treatment approach in knee osteoarthritis-A randomized controlled trial. Osteoarthritis Cartilage 2018; 26:620-630.

7. Qaseem A, Wilt TJ, McLean RM, Forciea MA. Noninvasive treatments for acute, subacute, and chronic low back pain: A Clinical Practice guideline from the American College of Physicians. Ann Intern Med 2017;166:514-530.

8. Bredesen DE, Amos EC, Canick J, et al. Reversal of cognitive decline in Alzheimer's disease. Aging 2016;8:1250-1258.

9. Cooley K, Szczurko O, Perri D, et al. Naturopathic care for anxiety: A randomized controlled trial ISRCTN78958974. PLoS One 2009;4:e6628.

10. Ritenbaugh C, Hammerschlag R, Calabrese C, et al. A pilot whole systems clinical trial of traditional Chinese medicine and naturopathic medicine for the treatment of temporomandibular disorders. J AlternComplement Med 2008;14: 475-487.

11. Hullender Rubin LE, Opsahl MS, Wiemer KE, et al. Impact of whole systems traditional Chinese medicine on in-vitro fertilization outcomes. Reprod Biomed Online 2015;30:602-612.

12. Rioux J, Thomson C, Howerter A. A pilot feasibility study of whole-systems ayurvedic medicine and yoga therapy for weight loss. Glob Adv Health Med 2014;3:28-35.

13. Wang C, Schmid CH, Iversen MD, et al. Comparative effectiveness of tai chi versus physical therapy for knee osteoarthritis: A randomized trial. Ann Intern Med 2016;165:77-86.

14. Selby JV, Beal AC, Frank L. The Patient-Centered Outcomes Research Institute (PCORI) national priorities for research and initial research agenda. JAMA 2012;307:1583-1584.

15. Porter Starr KN, McDonald SR, Bales CW. Obesity and physical frailty in older adults: A scoping review of lifestyle intervention trials. J Am Med Dir Assoc 2014;15:240-250.

16. Bradley R, Sherman KJ, Catz S, et al. Adjunctive naturopathic care for type 2 diabetes: Patient-reported and clinical outcomes after one year. BMC Complement Altern Med 2012;12:44.

17. Shalom-Sharabi I, Samuels N, Lavie O, et al. Effect of a patienttailored integrative medicine program on gastro-intestinal concerns and quality of life in patients with breast and gynecologic cancer. J Cancer Res Clin Oncol 2017;143:1243-1254.

18. Koithan M, Verhoef M, Bell IR, et al. The process of whole person healing: "unstuckness" and beyond. J Altern Complement Med 2007;13:659-668.

19. Oberg EB, Bradley R, Hsu C, et al. Patient-reported experiences with first-time naturopathic care for type 2 diabetes. PLoS One 2012; 7:e48549.

20. Ritenbaugh C, Nichter M, Nichter MA, et al. Developing a patient-centered outcome measure for complementary and alternative medicine therapies I: Defining content and format. BMC Complement Altern Med 2011;11:135.

21. Thompson JJ, Kelly KL, Ritenbaugh C, et al. Developing a patient-centered outcome measure for complementary and alternative medicine therapies II: Refining content validity through cognitive interviews. BMC Complement Altern Med 2011;11:136.

22. Hsu C, Bluespruce J, Sherman K, Cherkin D. Unanticipated benefits of CAM therapies for back pain: An exploration of patient experiences. J Altern Complement Med 2010;16: 157-163.
23. Johnson MB, Bertrand SW, Fermon B, Coleman JF. Pathways to healing: Person-centered responses to complementary services. Glob Adv Health Med 2014;3:8-16.

24. Proia KK, Thota AB, Njie GJ, et al. Team-based care and improved blood pressure control: A community guide systematic review. Am J Prev Med 2014;47:86-99.

25. Hamre HJ, Witt CM, Kienle GS, et al. Long-term outcomes of anthroposophic therapy for chronic low back pain: A two-year follow-up analysis. J Pain Res 2009;2:75-85.

26. Attias S, Keinan Boker L, Arnon Z, et al. Effectiveness of integrating individualized and generic complementary medicine treatments with standard care versus standard care alone for reducing preoperative anxiety. J Clin Anesth 2016;29:54-64.

27. Grant SJ, Bensoussan A. The process of care in integrative health care settings-A qualitative study of US practices. BMC Complement Altern Med 2014;14:410.

28. Penney LS, Ritenbaugh C, Elder C, et al. Primary care physicians, acupuncture and chiropractic clinicians, and chronic pain patients: A qualitative analysis of communication and care coordination patterns. BMC Complement Altern Med 2016;16:30.

29. DeBar LL, Elder C, Ritenbaugh C, et al. Acupuncture and chiropractic care for chronic pain in an integrated health plan: A mixed methods study. BMC Complement Altern Med 2011;11:118.

30. Herath C, Zhou Y, Gan Y, et al. A comparative study of interprofessional education in global health care: A systematic review. Medicine 2017;96:e7336.

31. Howe A, Smajdor A, Stockl A. Towards an understanding of resilience and its relevance to medical training. Med Educ 2012;46:349-356.

32. Hooking up: Don Berwick, integrative medicine and his call for a radical shift to "health creation." 2014. Online document at: https://www.huffpost.com/entry/don-berwickintegrative-m_b_4781105, accessed November 25, 2018.

33. American Hospital Association WM. Toward a healthier tomorrow. 2013. Online document at: https://www.hhnmag.com/ articles/5563-toward-a-healthier-tomorrow, accessed November 25, 2018.

34. Gate L, Warren-Gash C, Clarke A, et al. Promoting lifestyle behaviour change and well-being in hospital patients: A pilot study of an evidence-based psychological intervention. J Public Health (Oxf) 2016;38:e292-e300.

35. Witt CM, Ausserer O, Baier S, et al. Effectiveness of an additional individualized multi-component complementary medicine treatment on health-related quality of life in breast cancer patients: A pragmatic randomized trial. Breast Cancer Res Treat 2015;149:449-460.

36. Seely D, Szczurko O, Cooley K, et al. Naturopathic medicine for the prevention of cardiovascular disease: A randomized clinical trial. CMAJ 2013;185:E409-E416.

37. Mills PJ, Wilson KL, Pung MA, et al. The self-directed biological transformation initiative and well-being. J Altern Complement Med 2016;22:627-634.

Address correspondence to: Charles Elder, MD, MPH, FACP Kaiser Permanente Center for Health Research 3800 North Interstate Avenue Portland, OR 97227

E-mail: charles.elder@kpchr.org 